\title{
Infrared Intracavity Laser Absorption Spectrometer
}

\author{
G. Medhi ${ }^{\mathrm{a}}$, A. V. Muravjov ${ }^{\mathrm{b}}$, H. Saxena ${ }^{\mathrm{a}}$, J. W. Cleary ${ }^{\mathrm{a}}$, C. J. Fredricksen ${ }^{\mathrm{a}}$, R. E. Peale ${ }^{\mathrm{a}}$, O. Edwards ${ }^{\mathrm{b}}$ \\ ${ }^{\mathrm{a}}$ Department of Physics, University of Central Florida, Orlando, Florida 32816, USA \\ 'Zyberwear Inc., 2114 New Victor Rd., Ocoee, Florida 34761, USA
}

\begin{abstract}
A spectral sensing method with sufficient sensitivity to detect vapors of low vapor-pressure compounds such as explosives would have great promise for defense and security applications. An opportunity is Intracavity Laser Absorption Spectroscopy (ICLAS) at IR wavelengths. Our approach is based on multi-mode external-cavity quantum cascade lasers and a scanning Fabry-Perot spectrometer to analyze the laser mode spectrum in the presence of a narrow band intracavity absorber. This paper presents results of numerical solution of laser rate equations that support feasibility of kilometer effective active-cavity path lengths and sensitivity to concentrations of $10 \mathrm{ppb}$. This is comparable to the saturated vapor pressure of TNT. System design considerations and first experimental results are presented at 10 and $70 \mu \mathrm{m}$ wavelengths.
\end{abstract}

Keywords: Intracavity, quantum cascade laser, fabry-perot, infrared, spectroscopy.

\section{INTRODUCTION}

No spectral sensing method exists with sufficient sensitivity to detect vapors of low vapor-pressure compounds, such as explosives. The opportunity is for ultra-trace molecular vapor recognition by Intracavity Laser Absorption Spectroscopy (ICLAS) at Mid-IR (MIR, 3-5 $\mu \mathrm{m}$ wavelengths), Long-Wave IR (LWIR, 8-12 $\mu \mathrm{m}$ wavelengths) and Terahertz (THz 50-200 $\mu \mathrm{m}$ wavelengths). Objectives are to detect characteristic absorption signatures of ultra-trace vapors in the multi-mode emission spectra of external-cavity IR and $\mathrm{THz}$ quantum cascade lasers (QCL). We have conceived a compact field deployable system for IR and THz spectral sensing of vapors that will be simple, low-power, man portable, and composed of all off-the-shelf components. An advantage is the exploitation of an underutilized set of spectral signatures, many of them new to science, enabled by the unprecedented sensitivity. Benefits include the invention of a new spectral detection and analytic methodology for ultra-trace vapors. Such methodology will complement and will be faster and more sensitive than current methods. Applications include military and commercial screening for threat compounds and contraband having very low vapor pressures. Such compounds include explosives, chemical gases, biological aerosols, drugs, and banned or invasive plants or animals. Also, biomedical breath analysis and non-invasive law-enforcement searches are envisioned.

\section{EXPERIMENT AND RESULTS}

\subsection{Sensitivity Estimate}

The sensitivity limit for a QCL-based intracavity laser absorption spectrometer has been estimated by numerical solution of the laser rate equations, ${ }^{1}$

and

$$
\begin{gathered}
\frac{d M_{q}}{d t}=-\gamma M+B_{o} N\left(M_{q}+1\right)-\kappa_{q} c M_{q} \\
\frac{d N}{d t}=P-A N-N \sum B_{q} M_{q}
\end{gathered}
$$

where $\lambda$ is the broad band cavity loss, $B_{o}$ the peak gain, $B_{q}$ the gain at the frequency of mode $q, \kappa_{q}$ the absorption coefficient of intracavity absorption at the $q^{\text {th }}$ axial laser mode, $c$ the light speed, $P$ the pump rate, and $A$ the 
spontaneous decay rate of the upper laser level. These equations characterize the regimes of QCL laser build-up, namely the laser inversion $N$, the total photon number $M$, the photon numbers $M_{q}$ in individual laser modes, and the final stability of the laser spectral output.
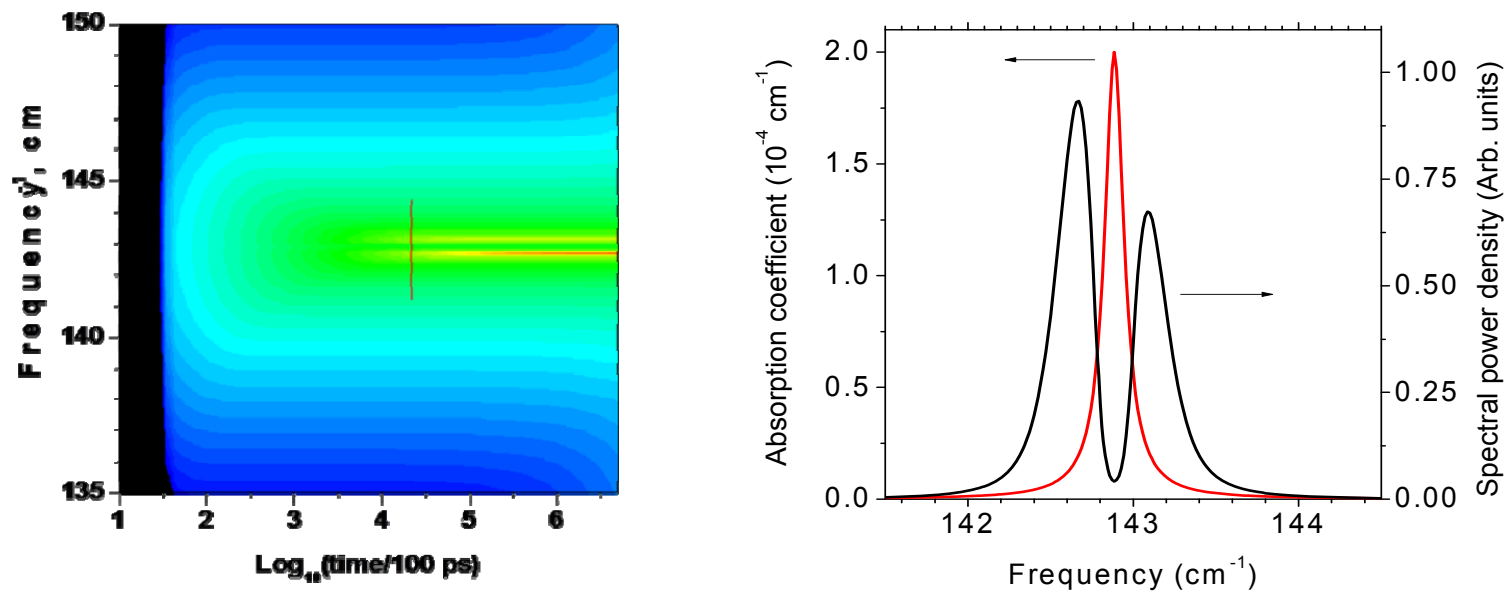

Figure 1. (Left) Time integrated laser emission spectrum. (Right) Weak intracavity absorption profile and its effect on the laser emission spectrum at $2 \mu$ s integration time

The numerical solution of Eqs. (1) and (2) can be presented as a time-integrated laser emission spectrum, as shown in Fig. 1 (left), where a weak intracavity absorption line has been included. The left figure displays the laser emission frequency corresponding to one of our THz QCLs. The logarithmic abscissa in the left hand figure represents the integration time, which can be interpreted as the laser-pulse duration. The signal strength is indicated by a logarithmic color scale with blue being few photons and red being many. The red vertical stripe at $2 \mu$ s corresponds to a 600 meter effective path. Fig. 1 (right) presents both the intracavity absorber line profile, having the very low peak value of just $2 \times 10^{-4} \mathrm{~cm}^{-1}$ and the laser emission profile. In a conventional transmittance experiment using a $10 \mathrm{~cm}$ vapor cell, the absorption dip in the intensity would have been only $0.2 \%$, which would be lost in the noise. In contrast, the ICLAS simulation reveals a nearly $100 \%$ deep absorption feature, which has been achieved due to the extraordinarily long intracavity path length.

Similar simulations for typical THz QCL parameters with $2 \mu$ s pulse duration suggest that absorptions as weak as $10^{-6} \mathrm{~cm}^{-1}$ might be detected. Infrared molecular absorption cross sections can be of order $10^{-18} \mathrm{~cm}^{2}$, which indicates a detection limit of $10^{12}$ molecules per cubic centimeter. In comparison with the number density of usual atmospheric molecules at standard conditions, this corresponds to 40 parts per billion. The saturated vapor pressure of TNT is $10^{-3}$ $\mathrm{Pa}, 13 \mathrm{ppb}$, or $3 \times 10^{11}$ molecules per cubic centimeter. In other words, the predicted sensitivity for a relatively short THz QCL pulse is within a factor of 3 needed to detect TNT in a confined space such as a shipping container. The needed increase can be achieved by increasing the pulse duration, which might easily be achieved by operating the laser closer to threshold, or by operating at a temperature slightly below $77 \mathrm{~K}$, as might be achieved using a Stirling cooler. We note that LWIR QCLs may operate CW at $77 \mathrm{~K}$, and would have substantially higher sensitivity.

A Fabry-Perot (FP) spectrum analyzer concept is shown conceptually in Fig. 2 (left). A THz QCL is housed in a liquid nitrogen cryostat, and its emission is collimated with an off-axis parabolic mirror and sent out through the cryostat window to the $95 \%$ reflecting cavity mirror. Target vapors pass through the open portion of the cavity. The $5 \%$ of the beam transmitted through the cavity mirror then passes a scanning central-spot FP interferometer and is collected by a detector. Calculated FP transmission as a function of FP gap for the laser spectrum of Fig. 1 (right) is presented in Fig. 2 (right), assuming an achievable THz FP finesse of 100. A near perfect representation of the laser spectrum of Fig. 1 is demonstrated. Note that for LWIR and MIR QCLs, no cryostat will be necessary. 

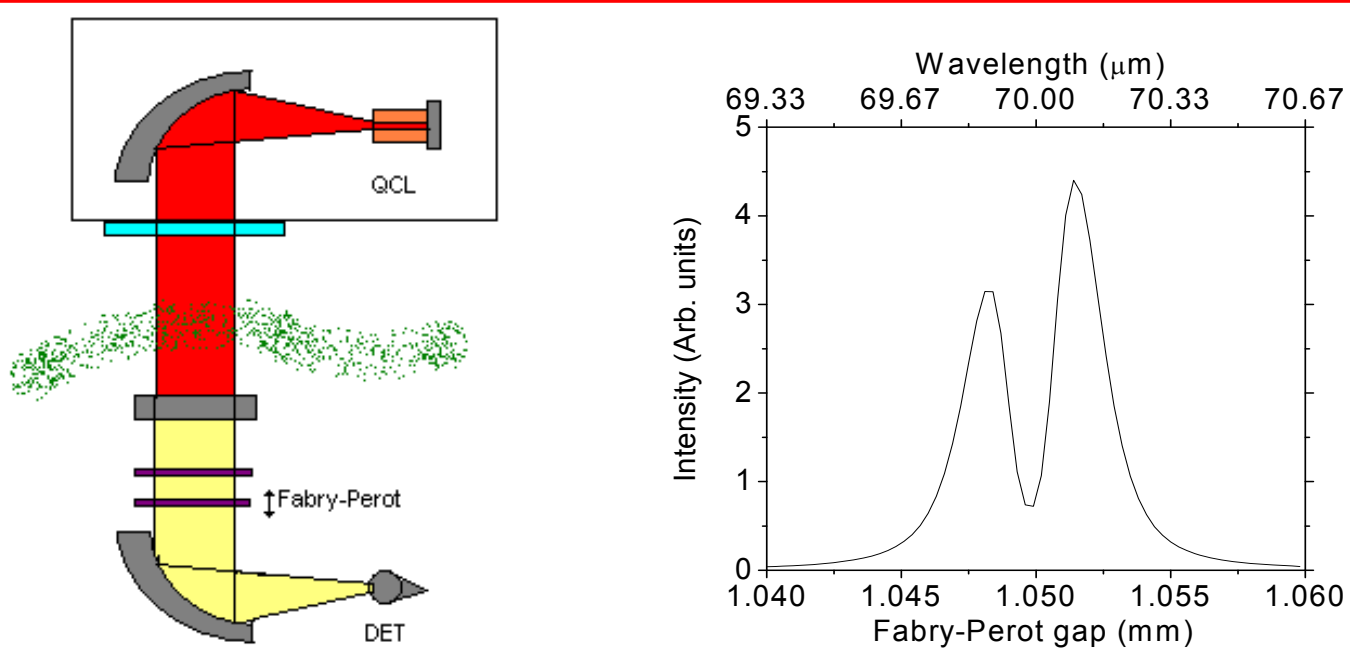

Figure 2. (Left) Schematic of QCL-based ICLAS system that uses a Fabry-Perot spectrometer to interrogate the laser spectrum. A vapor cloud passes inside the laser cavity. (Right) Recorded emission spectrum as a function of FP gap, which is directly convertible to wavelength as shown on the top axis.

To collect the spectrum of Fig. 2, where $30 \mu \mathrm{m}$ of mirror travel is required in $300 \mathrm{~nm}$ steps, 100 laser shots are required. Each laser shot requires about $60 \mu \mathrm{J}$ of electrical power, giving a requirement for the power supply of $6 \mathrm{~mJ}$ per spectrum. For comparison, a single $9 \mathrm{~V}$ battery stores $16,000 \mathrm{~J}$ of energy. Thus, battery operation is feasible, especially in the LWIR where the QCL requires no cryocooler.

\subsection{Terahertz}

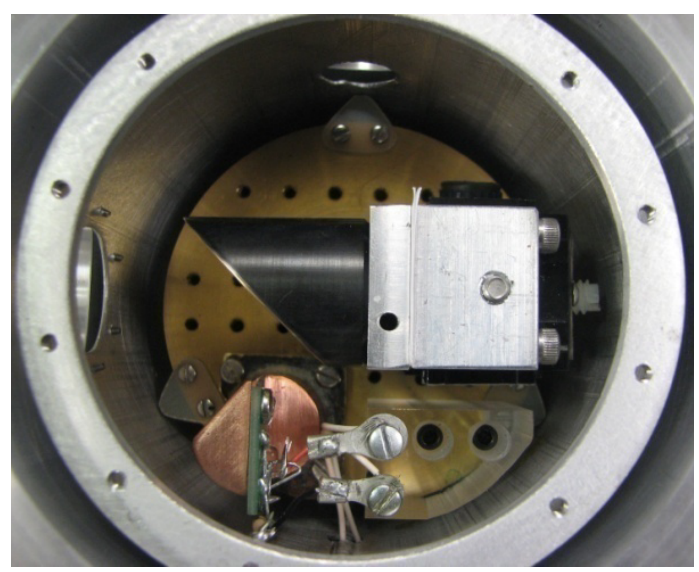

Figure 3. Mount and collimator for cryogenic external cavity for THz QCL.

Fig. 3 presents a photograph of the cryostat-mounted optics that were designed and fabricated for the THz QCL purchased from TRION. The laser was cooled to $77 \mathrm{~K}$ and excited by a laser diode driver with $10 \mu \mathrm{s} \times 1.4 \mathrm{~A}$ pulses at $20 \mathrm{~Hz}$ rep rate. The DEI laser diode driver is externally triggered by a Stanford DG535 pulse generator operating in burst mode. Fifty pulses at $1 \mathrm{kHz}$ rep rate, followed by $50 \mathrm{~ms}$ of no triggers achieves the $50 \%$ slow chopping needed for the Golay. The laser pulse duration is varied from 1 to $10 \mu \mathrm{s}$, giving an excitation duty cycle from 0.1 to $1 \%$. The collimated laser beam was collected outside the $25-\mu \mathrm{m}$-thick Mylar cryostat window by an off-axis parabola and focused onto the entrance aperture of a Golay cell (a sensitive room temperature bolometer). The Golay output was synchronously lock-in amplified. Measurements of lasing threshold are presented in Fig. 4 (left). The trace for $10 \mu \mathrm{s}$ 
pulse duration ( $1 \%$ duty cycle) falls below the $5 \mu$ s trace because the laser overheats. The threshold is about $0.95 \mathrm{~A}$ independent of pulse duration. At high current, the power tends to fall for the long pulses, again due to overheating.
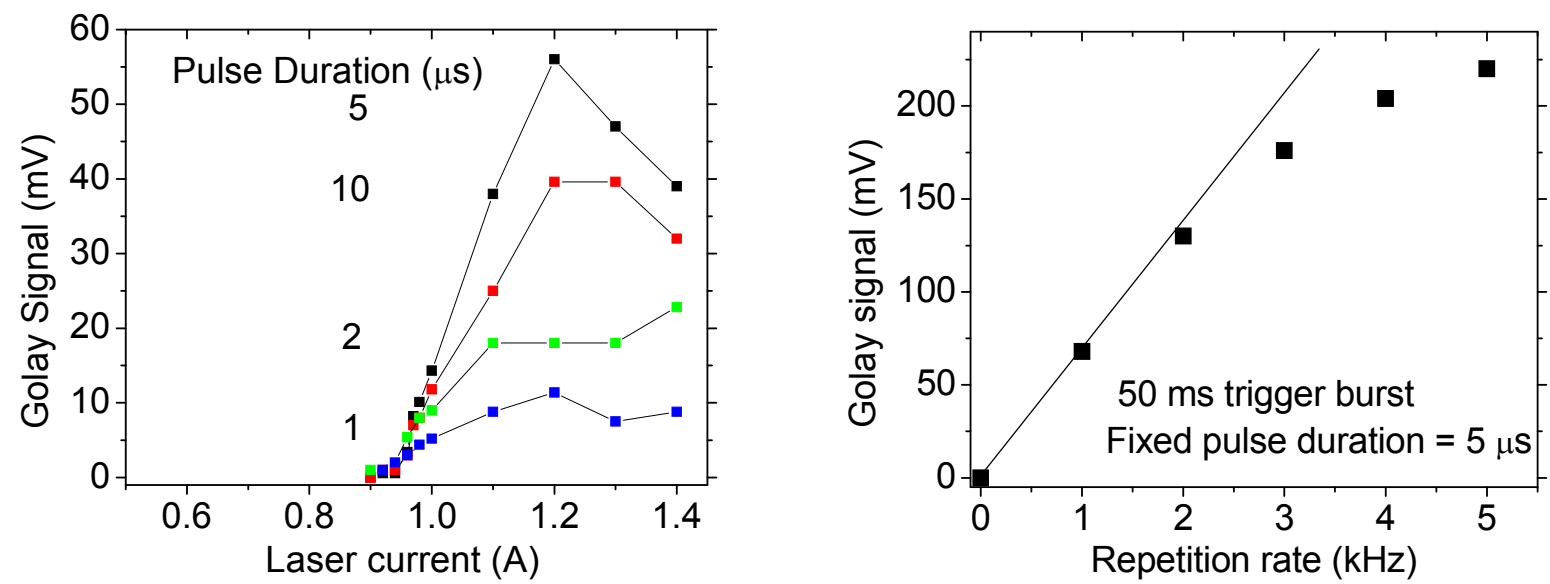

Figure 4. (Left) Laser emission signal as a function of laser current for 4 different pulse durations. (Right) Golay signal from the TRION THz QCL vs repetition rate, keeping the trigger burst parameters constant (50 ms of triggers followed by $50 \mathrm{~ms}$ of none) for constant $5 \mu$ s pulse duration.

Fig. 4 (right) more clearly demonstrates the overheating effect. The duty cycles were increased through the values $0.5,1.0,1.5,2.0$, and $2.5 \%$. The roll-off indicates overheating, making clear the specified $3 \%$ duty limit. From the maximum achieved Golay signal, the Golay responsivity, the duty cycle, and pulse duration we obtain for the QCL an average power $=6.7 \mu \mathrm{W}$, peak power $=0.27 \mathrm{~mW}$, and pulse energy $=1.3 \mathrm{~nJ}$. Accounting for atmospheric absorption (see below), we multiply these figures by 2 or more to get actual emission power at the laser end facet. This result is in reasonable agreement with TRION specifications.
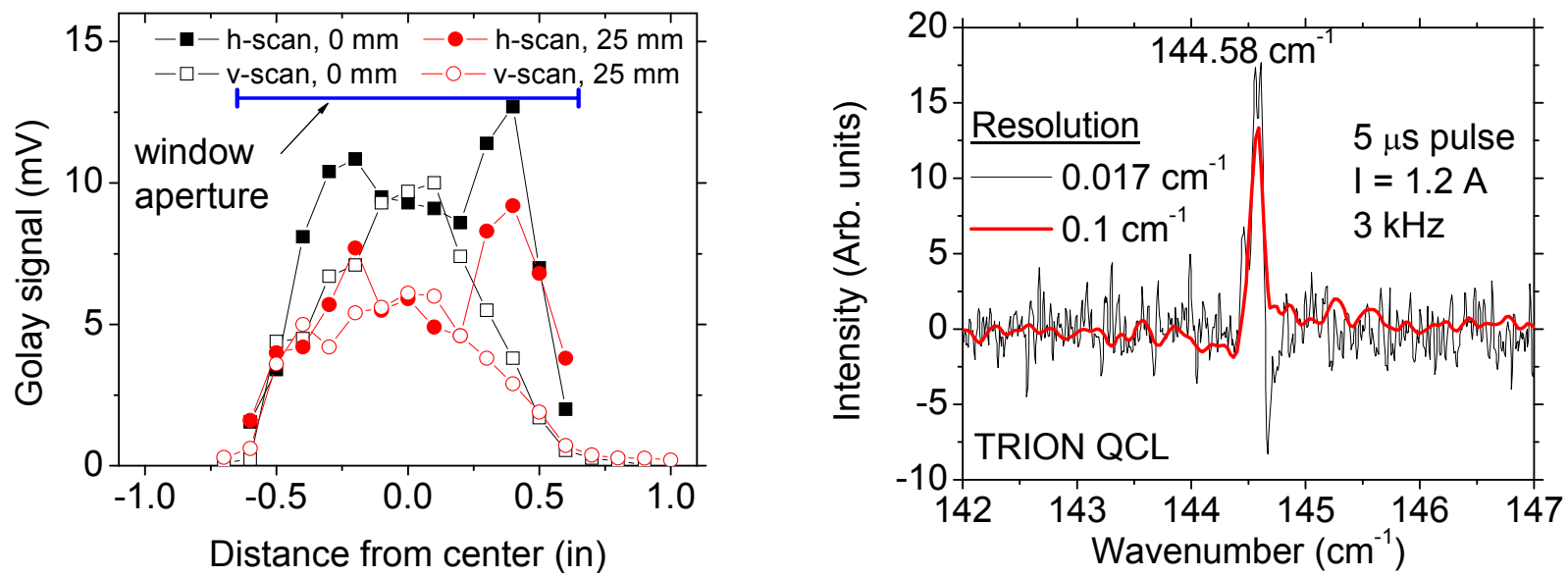

Figure 5. (Left) TRION THz QCL vertical and horizontal beam profiles as a function of distance from cryostat window. (Right) High resolution emission spectrum of TRION THz QCL.

Fig. 5 (left) presents measured beam profiles and the effect of atmospheric attenuation. The beam has a double peak in the horizontal scan and falls off more smoothly from the center in the vertical scan. The width of the profile is a bit less than the diameter of the output window and is probably defined primarily by the cold shield aperture diameter. Moving the detector back from the cryostat by $2.5 \mathrm{~cm}$ mainly decreases the intensity by the factor 0.63 with little beam divergence observed. From this is determined the absorption coefficient of the laboratory air at the specified $70 \mu \mathrm{m}$ laser 
wavelength as $0.19 \mathrm{~cm}^{-1}$ or $0.81 \mathrm{db} / \mathrm{cm}$. Half of the power is lost in a distance of $3.7 \mathrm{~cm}$. Nevertheless, even with no collection optic, the Golay can still detect laser signal at a distance of 0.5 meter.

A high resolution emission spectrum (Fig. 5, right) was collected using a Fourier spectrometer (Bomem DA8). The laser line width is seen to be $0.1 \mathrm{~cm}^{-1}$, due probably to temperature-induced shift during the laser pulse. Single mode emission is undesirable for ICLAS, so we need to operate the laser closer to threshold, at lower current and shorter pulse duration. The spectrum, taken with a room temperature DTGS pyroelectric detector, has good signal-to-noise ratio. For our project, it shows that a simple, inexpensive, room temperature pyroelectric detector can be used instead of a Golay or $4 \mathrm{~K}$ bolometer.

After the external cavity laser, the next required enabling technology for a THz ICLAS system is a highresolution real-time means of monitoring the emission spectrum. We consider a scanning central-fringe Fabry-Perot interferometer to be the most attractive option. To operate at $70 \mu \mathrm{m}$ wavelength requires a minimum FP translation of at least $35 \mu \mathrm{m}$ to catch at least one resonance. We have implemented a translation stage as shown in the photograph of Fig. 6 (left). The system is controlled by Labview, and the DC output of the lock-in is recorded as a function of stage position.

Fig. 6 (right) presents the resonances obtained using the QCL with $69.6 \mu \mathrm{m}$ wavelength and DSP silicon wafers as the FP mirrors. The expected reflectivity for these mirrors is only $R=[(n-1) /(n+1)]^{2}=30.0 \%$. The measured finesse $=$ $\lambda / 2 F W H M=2.5=\pi \sqrt{R} /(1-R)$ gives $\mathrm{R}=31 \%$, in very good agreement with expectations. Metal mesh mirrors with higher reflectivity will increase the finesse and resolving power of this instrument.
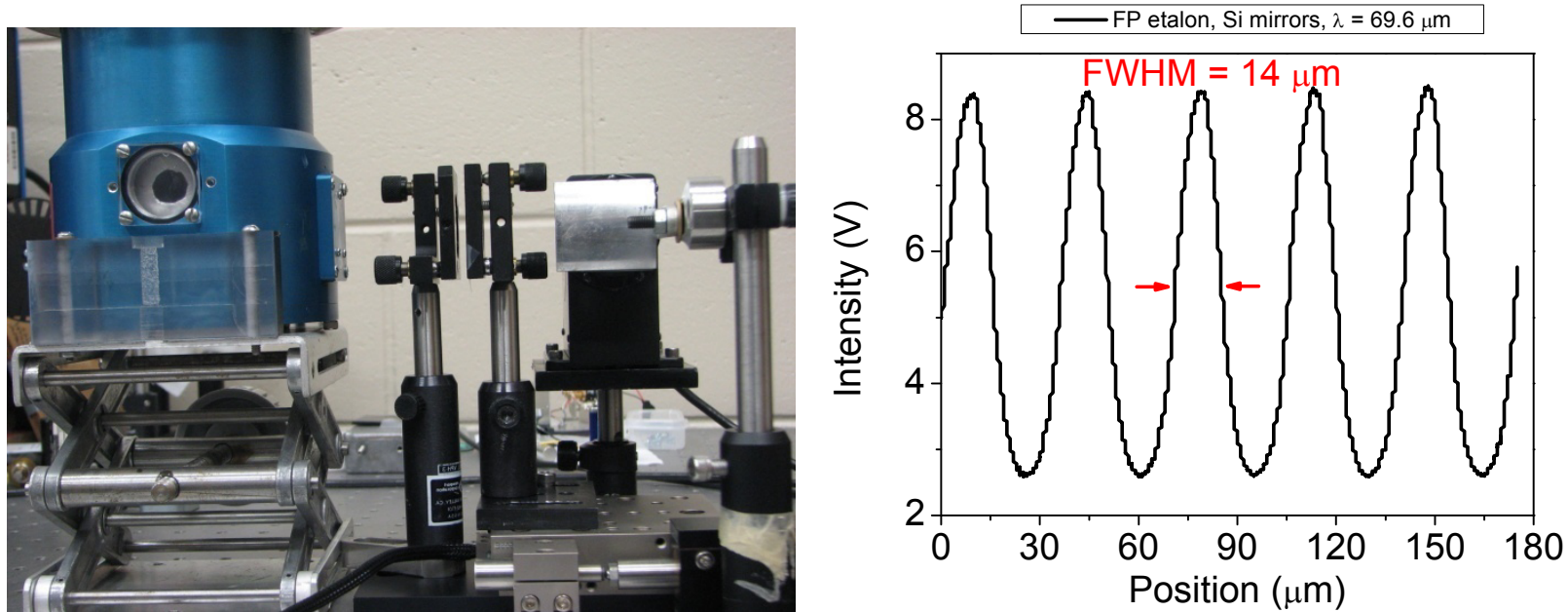

Figure 6. (Left) THz Fabry-Perot set up. From left to right, the THz QCL is mounted in the cryostat with internal collimating optics and a polyethylene window. A fixed mirror faces a moving mirror which is mounted to a motorized precision translation stage. An off-axes parabolic mirror (aluminum) focuses the transmitted radiation to a golay cell detector (black). (Right) Fabry-Perot spectrum of THz QCL.

Implementation of a THz QCL-based ICLAS system is clearly complicated by the requirement for a cryostat to house the laser. This greatly restricts the working space and the cryostat window introduces a loss element into the cavity. Moreover, emission spectra of target vapor molecules are poorly known in the $\mathrm{THz}$, there is very strong interference from water vapor, and the molecular absorption cross sections for the type of vibration likely to occur in the $\mathrm{THz}$ (libration) are very low due to small dipole moments for such low frequency motions. ${ }^{2}$ The long-wave IR ( $\sim 30$ $\mathrm{THz}$ ) is a much more attractive sensing space, and we turn our attention to this spectral range next.

\subsection{Long-wave IR}

Fig. 7 (left) presents a schematic of a stable confocal cavity consisting of two $90^{\circ}$ off axis gold coated parabolic mirrors of $2.5 \mathrm{~cm}$ focal length and two flat mirrors placed at a distances of $\mathrm{L}_{1}$ and $\mathrm{L}_{4}$ from the optical center of the parabolic mirrors. The QCL was placed at the common focal point of the two parabolic mirrors. A He-Ne laser was used 
to align the whole system before inserting the QCL. A small hole of diameter $0.15 \mathrm{~cm}$ in the middle of the first flat mirror outcoupled laser emission from the cavity into the spectrometer. A photograph of the experimental set up is shown in Fig. 7 (right).
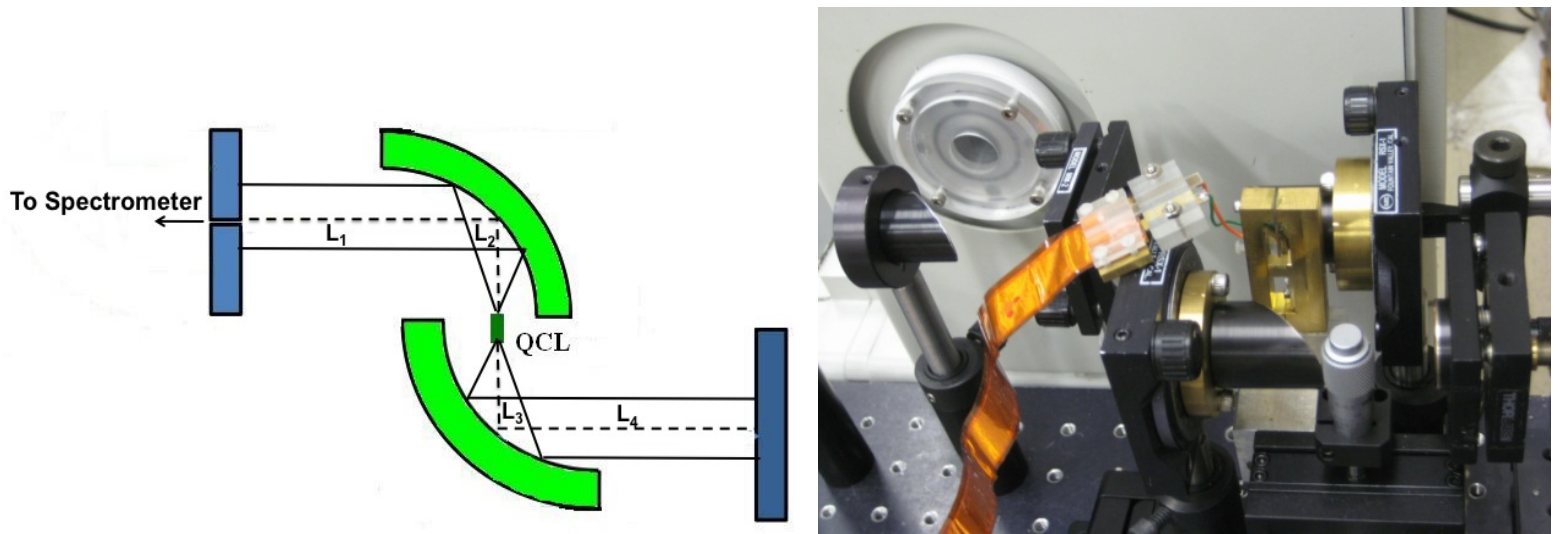

Figure 7. (Left) Schematic of the current confocal external cavity. (Right) Photograph of external cavity QCL for LWIR The QCL is housed in the rectangular brass holder. A parabolic mirror and out-coupling flat mirror are mostly obscured by the laser mount and flat excitation cable, respectively. A third parabola directs the emission into the spectrometer through a $\mathrm{NaCl}$ vacuum window.

Fig. 8 (left) presents the overall multimode laser emission spectrum of the LWIR QCL measured with the Fourier spectrometer. The spectral emission width spans $60 \mathrm{~cm}^{-1}$. The longitudinal mode separation of $\sim 1 \mathrm{~cm}^{-1}$ is defined by the distance between the end facets of the QCL and its refractive index. Fig. 8 (right) presents high resolution spectra of the laser emission with and without the external cavity. With the external cavity, a mode fine structure appears indicating the successful implementation of the external cavity.
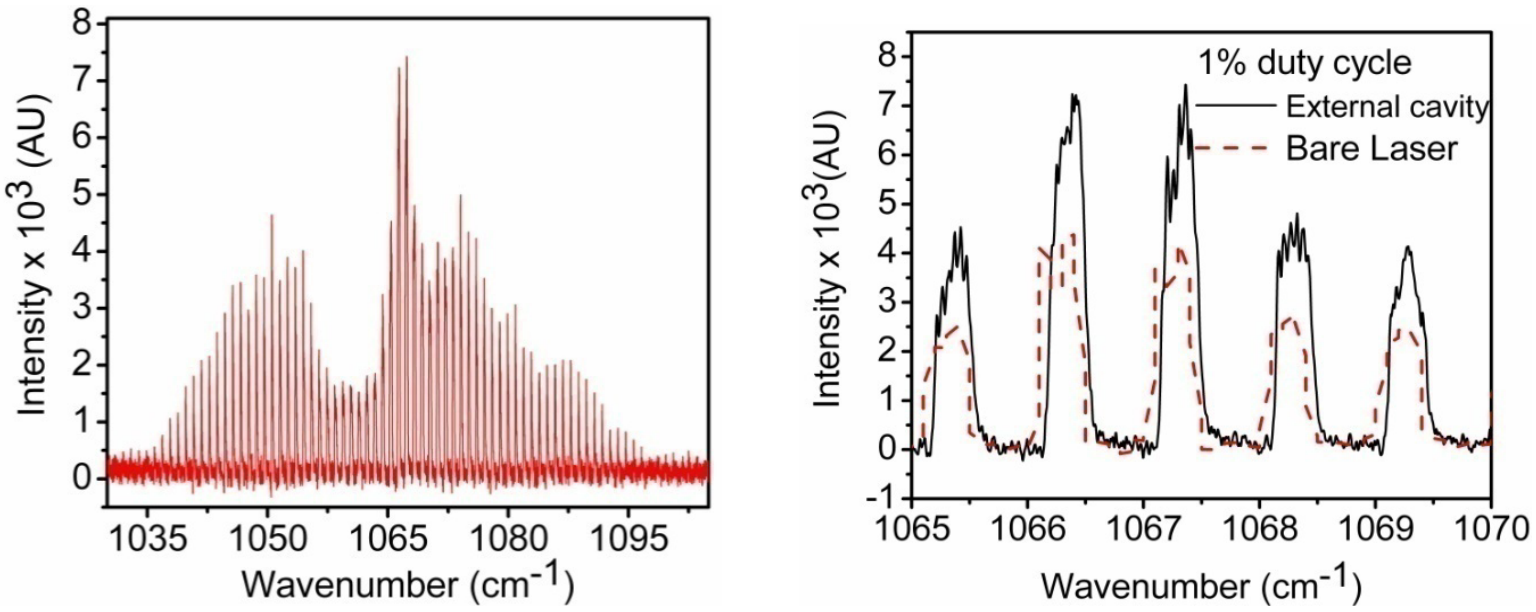

Figure 8. (Left) Entire multimode QCL emission spectrum. (Right) Close up of emission spectrum with and without the external cavity.

Closer inspection of the mode fine structure is presented in Fig. 9, which reveals a mode spacing of $\sim 0.05 \mathrm{~cm}^{-1}$. In the Fig. 7 (left) schematic, we have $\mathrm{L}_{1}=6.95 \mathrm{~cm}, \mathrm{~L}_{2}=\mathrm{L}_{3}=2.5 \mathrm{~cm}, \mathrm{~L}_{4}=6.75 \mathrm{~cm}$. A mode separation of $0.05 \mathrm{~cm}^{-1}$ corresponds to a total cavity length of $\sim 9.25 \mathrm{~cm}$, which is just the $\mathrm{L}_{3}+\mathrm{L}_{4}$ half of the external cavity length. For the ICLAS application, it is necessary to fill the entire spectral space of interest with external cavity modes. We see in Figs. 8-9 that this goal is impeded by the mode structure of the active crystal itself. Thus, we will require anti-reflection coatings on the end facets of the laser or a laser with end facets cut and polished at Brewster's angle. 


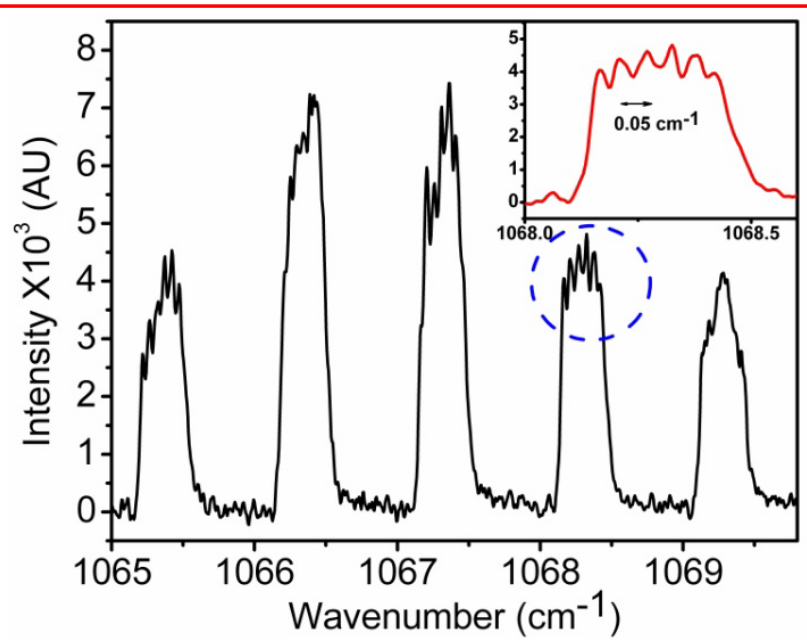

Figure 9. High resolution spectrum of external cavity laser modes. The inset demonstrates a mode spacing of $0.05 \mathrm{~cm}^{-1}$, which corresponds to a cavity length of $\sim 9.25 \mathrm{~cm}$.

Next in the level of importance to the ICLAS device concept is a compact means of determining the laser spectrum in real time. We have selected a high-resolution Fabry-Perot Spectrometer for this purpose. Fig. 10 (left) presents a photograph of a prototype FP spectrometer based on a piezo-cylinder to control the mirror movement.
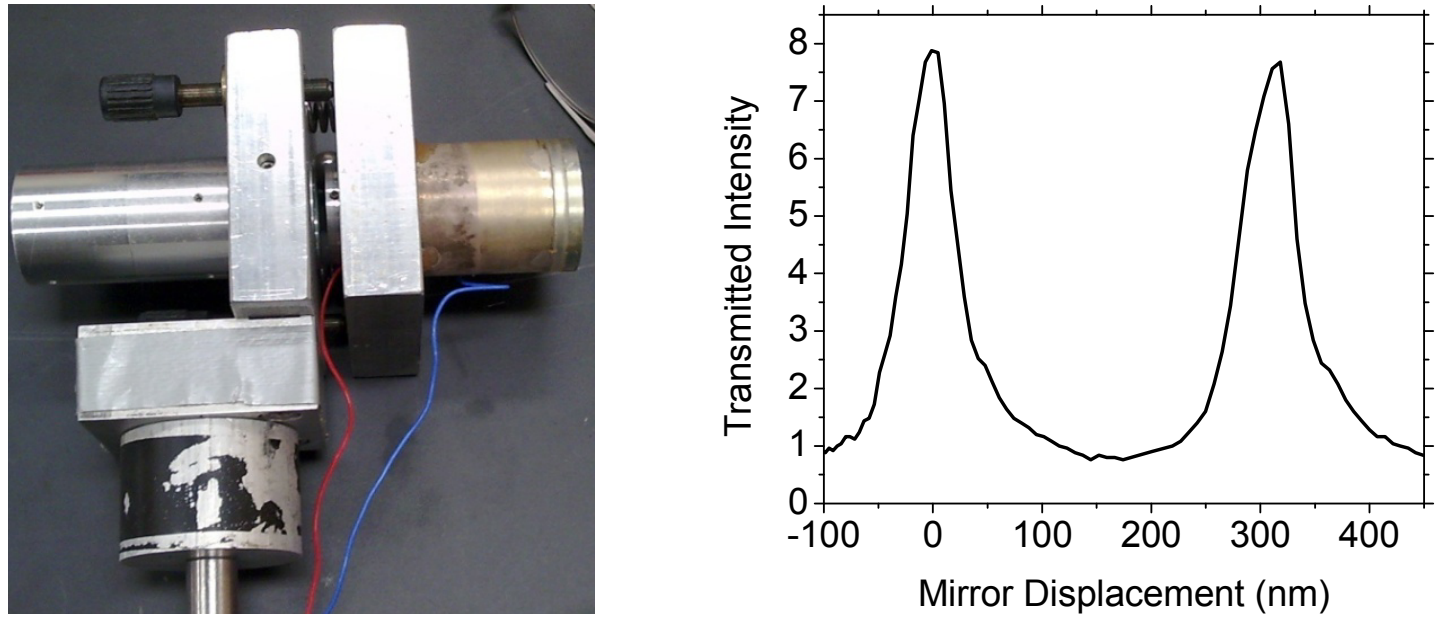

Figure 10. (Left) photograph of Fabry-Perot spectrometer for the long-wave IR. (Right). Transmission resonances measured using dielectric mirrors and a HeNe laser

The spectrometer has been tested using dielectric mirrors with $98 \%$ reflectivity at $\mathrm{HeNe}$ laser wavelength. The piezo was simply plugged into line voltage via an isolation transformer, and the transmittance resonances observed in real-time on an oscilloscope. Rough alignment is achieved by measuring the space between the four corners of the holder with calipers. Then, final alignment is achieved by adjusting the screws until the sharpest resonances are observed on the scope. The nominal finesse for the given mirrors should have been 155 . The actual finesse achieved was 6, showing that there is still improvement to be gained in the alignment. The translation achieved with $170 \mathrm{~V}$ AC peak-to-peak applied was several tenths of a micron. Thus, the translation of this first system is insufficient for what we estimate is needed at our long-wave IR wavelength $(\sim 5 \mu \mathrm{m}$, see below). However, it is a good first demonstration, and lessons learned, with different piezos and higher voltages, will lead to success.

We require the finesse to be highest at our $9.4 \mu \mathrm{m}$ wavelength. The Free Spectral Range (FSR) must exceed $0.222 \mu \mathrm{m}$. A mirror separation of $198 \mu \mathrm{m}$ must be tunable over $5 \mu \mathrm{m}$. The resolving power necessary to observe vapor 
lines is $Q=\lambda / \Delta \lambda=5222$. This implies finesse better than 124 , or a reflectivity for the FP mirrors of $97.5 \%$. These specifications were arrived at as follows. We need to be able to move the FP transmission resonance over the emission bandwidth of our Quantum Cascade Laser. That range is 9.524 to $9.302 \mu \mathrm{m}$ wavelength. The FSR therefore has to be at least $0.222 \mu \mathrm{m}$. That implies a maximum resonance order of about $\mathrm{k}=\lambda / F S R=42$, or a maximum mirror separation $=$ $k \lambda / 2=0.198 \mathrm{~mm}$. This mirror separation has to be tuned over $d x=k^{d \lambda / 2}=42 *(0.222) / 2=4.7 \mu \mathrm{m}$ in order to cover the full FSR. The feature we are looking for in that range is expected to have a minimum linewidth of about $0.2 \mathrm{~cm}^{-1}$, or $1.8 \mathrm{~nm}$ at our center wavelength. The necessary resolving power is $\mathrm{Q}=9400 \mathrm{~nm} / 1.8 \mathrm{~nm}=5222$. Given the maximum resonance order, this implies a required minimum finesse of about $F=Q / k=124$. Reflectivity of the FP mirrors, from $F=\pi \sqrt{R} /(1-R)$, gives $\mathrm{R}=97.5 \%$, which is needed at $9.4 \mu \mathrm{m} \pm 0.1 \mu \mathrm{m}$ wavelength, i.e. $1 \%$ bandwidth. Wedged $\mathrm{ZnSe}$ mirrors that will fulfill our requirements are being procured from MolTech $\mathrm{GmbH}$ Berlin. These are AR coated at 9.3-9.5 $\mu \mathrm{m}$ on one side only, while the other side is HR coated with reflectivity $>97.5 \%$.

\section{SUMMARY}

The key enabling technology for a THz-LWIR ICLAS system is the quantum cascade laser with external cavity. Second in importance is the Fabry-Perot spectrometer to measure the multi-mode emission spectrum at high resolution. Progress on both was reported here. An external cavity was clearly demonstrated in the LWIR. Fabry-Perot systems for both LWIR and THz were defined and demonstrated with a clear path forward to improvement.

\section{ACKNOWLEDGMENTS}

This project is supported by an Army Phase II SBIR (Dwight Woolard, PM).

\section{REFERENCES}

[1] V. M. Baev, T. Latz, and P. E. Toschek, "Laser intracavity absorption spectroscopy,” Appl. Phys. B 69, 171-202 (1999).

[2] R. E. Peale, A. V. Muravjov, C. J. Fredricksen, G. D. Boreman, H. Saxena, G. Braunstein, V. L. Vaks, A. V. Maslovsky, S. D. Nikifirov "Spectral signatures of acetone from ultraviolet to millimeter wavelengths," Proc. 2006 Intl. Symp. Spectral Sensing Research, May 29 to Jun 2, 2006, Bar Harbor, Maine. 